\title{
Evaluation and comparison of three IR detectors and three amplifier designs for a new, high-speed IR pyrometer
}

\author{
J.A. Young ${ }^{* a}$, S. Borror ${ }^{\mathrm{a}}$, A.W. Obst ${ }^{\mathrm{b}}$, J.R. Payton ${ }^{\mathrm{b}}$, A. Seifter ${ }^{\mathrm{b}}$ \\ ${ }^{a}$ Bechtel Nevada, Los Alamos Operations, Los Alamos, NM 87544 \\ ${ }^{b}$ Los Alamos National Laboratory, Physics Division, Los Alamos, NM 87545
}

\begin{abstract}
At Los Alamos National Laboratory (LANL), a high-speed, four-wavelength, infrared (IR) pyrometer has been used for surface temperature measurements in shock-physics experiments for several years. The pyrometer uses solidstate detectors and a single fiber-optic cable for transmission of light from the target surface to the detectors. This instrument has recently been redesigned for an upcoming experiment at the Nevada Test Site (NTS). Three different IR detectors (two $\mathrm{HgCdTe}$ variants as well as the existing InSb chip) were compared for sensitivity, signal-to-noise ratio, and bandwidth. Of major concern was detector amplifier recovery time from overload saturation. In shockphysics experiments, a short but very bright precursor frequently accompanies shock breakout (often from trapped air). This precursor can saturate the amplifier and may "swamp-out" the signal of interest before the amplifier recovers. With this in mind, we evaluated two new amplifier designs by the Perry Amplifier Company for linearity, signal-to-noise characteristics, gain, and saturation recovery time. This paper describes experimental setup for detector comparison and results obtained. Furthermore, we discuss new amplifier design and suitability for highspeed infrared pyrometry in shock physics experiments.
\end{abstract}

Keywords: pyrometer, IR detector, $\mathrm{HgCdTe}$, InSb, shock physics

\section{INTRODUCTION}

Los Alamos National Laboratory routinely performs shock physics experiments to constrain the equation-of-state (EOS) for different materials. Aside from pressure, temperature is one of the most important parameters measured in these experiments. Efforts are also underway to measure the temperature at the Hugoniot pressure region inside a target sample. ${ }^{1}$ Previously pyrometry was the only well-established way to determine temperatures in shock physics experiments.

Several years ago, a solid-state, four-channel, single-fiber, high-speed, IR pyrometer was developed and fielded at LANL's Physics Division. ${ }^{2}$ However, for an upcoming experiment at the NTS, this instrument must be redesigned to be smaller overall (due to space constraints) and to avoid using liquid nitrogen to cool the detectors (due to the instrument's inaccessibility for long periods before the experiment). As dictated by the scope of the design parameters, we sought the latest technology in infrared detectors and fast amplifiers. (The temperatures of interest are in the $500-1000 \mathrm{~K}$ region, which corresponds to infrared wavelengths in the $2-$ to 5 - $\mu \mathrm{m}$ region. For more details on pyrometry, see DeWitt and Nutter. ${ }^{3}$ )

\subsection{Detector requirements}

To be considered as a high-speed pyrometer for shock physics applications, detectors must fulfill the following requirements:

- High speed: Due to the short duration (hundreds of nanoseconds to several microseconds) and fast signal rise-time (nanoseconds) of shock physics experiments, detector bandwidth should be as high as possible.

*youngja@nv.doe.gov; phone 1505 663-2025; fax 1505 663-2105 
- Linearity: In order to calculate a radiance temperature from the measured pyrometer signal, the detector/amplifier combination should be linear over the full dynamic range. Otherwise, the pyrometer must be calibrated in a very time-consuming way, thus greatly complicating data reduction.

- High sensitivity: As mentioned above, temperatures of interest are sometimes as low as 500K. Surfaces at this temperature emit thermal radiation of very low intensity. Detecting thermal radiation at these temperatures requires high detector sensitivity.

- Low noise: Detector noise contributes the highest uncertainty at low temperatures; hence, a high signal-to-noise ratio is desirable for pyrometric detectors used in low-temperature applications.

- High dynamic range: The thermal radiation emitted by hot surfaces can be described by Planck's law. This law shows that radiance increases exponentially with (absolute) temperature. Only if the dynamic ranges of the detectors and amplifiers are sufficiently high can a reasonable temperature range be covered.

- Reproducibility/thermal stability: To determine the temperature of a hot surface, the emitted radiance is compared to the radiance of a surface at a known temperature (a blackbody radiator). This calibration is often very difficult and time-consuming, and it can usually only be performed once for each experimental series, which often lasts for several weeks. Conditions (mainly the temperature of the environment in which experiments are conducted) can change over this period. Therefore, the detectors need low temperature dependence and high temporal stability.

\subsection{Amplifier requirements}

For the same reasons mentioned above, the amplifiers must be fast (high bandwidth), low-noise, linear, and able to perform over a high dynamic range. An additional problem in shock physics experiments is that at the time of shock breakout, a short (some 10- to 100-ns), bright flash can occur, as shown in Figure 1. (These flashes are most commonly caused by air lighting up, surface contamination, jets at target edges, or ejecta.)

Sometimes these flashes are bright enough to drive the amplifier and/or detector into saturation, which can lead to the charging of internal capacitance with a relatively large time constant (up to several $\mu$ s). In this case, the experiment is over before the amplifier recovers from this overload situation (see Figure 2), and no meaningful data is recovered.

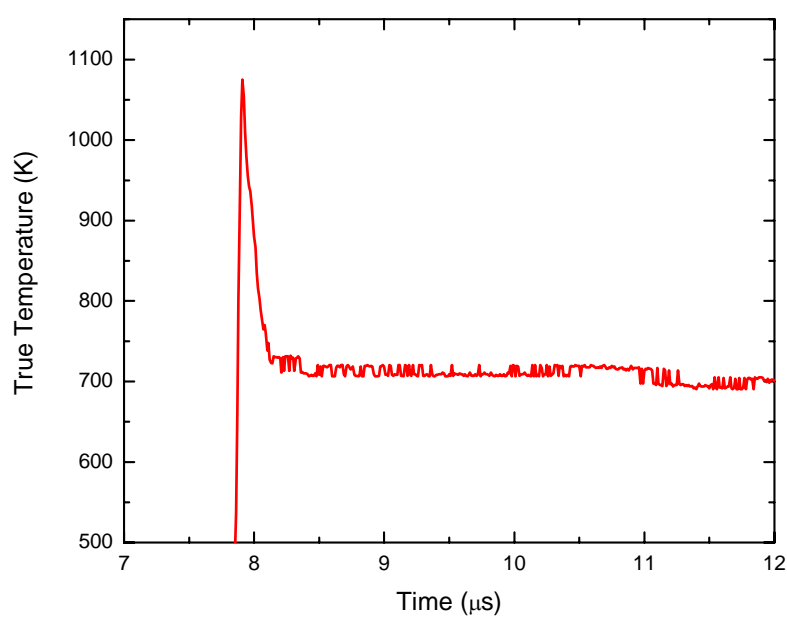

Fig. 1. Shock breakout peak at a direct-driven, highexplosive experiment on tin. ${ }^{4}$

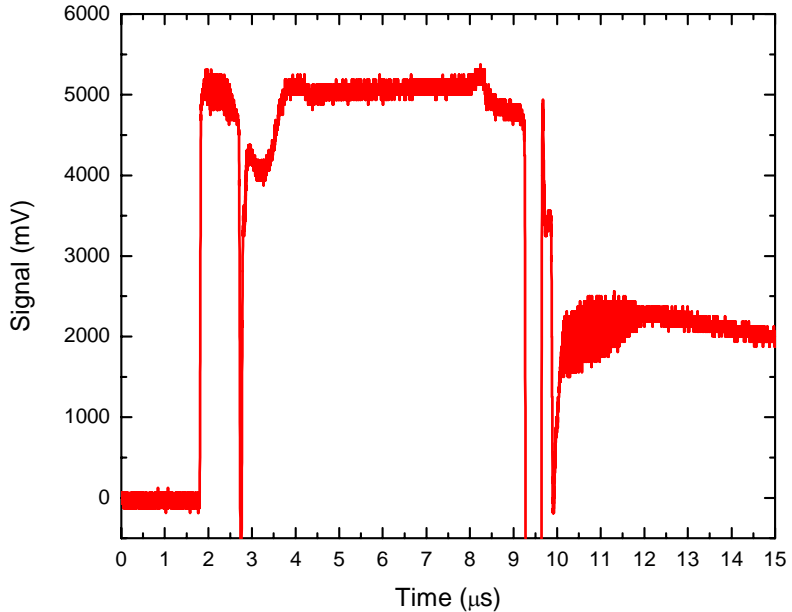

Fig. 2. Amplifier saturation from a powder gun experiment on molybdenum. 
Shock physics experiment designers attempt to eliminate the cause of these flashes at shock breakout. As this cannot be accomplished every time, amplifiers should be designed to recover within a reasonable time (depending on experiment duration) from these overload conditions.

\section{COMPARISON METHODOLOGY}

\subsection{Detector comparison}

The existing pyrometer uses indium antimonide (InSb) detectors from Judson Technology, Inc. ${ }^{5}$ The InSb chips have 1-mm-diameter circular active areas housed in an evacuated environment. The housings themselves are contained within liquid nitrogen dewars used to maintain a constant temperature of $77 \mathrm{~K}$, to keep "dark noise" at a minimum. A broadband antireflective-coated silicon window on the dewar is used in front of the detector to filter visible background light. These detectors were compared to a mercury-cadmium-telluride (MCT) detector from Fermionics $^{6}$ and another MCT detector from Kolmar Technologies, Inc., ${ }^{7}$ borrowed from Daniel Dolan at Sandia National Laboratories.

The detector comparison began with the setup shown in Figure 3. In order to preserve the emissive characteristics of the blackbody source and to avoid chromatic aberration, off-axis parabolic mirrors were used to focus the radiant energy onto the detectors' active areas (underfilled). A chopper wheel provided an amplitude contrast of detector output. The blackbody temperature was stepped up from $100^{\circ} \mathrm{C}$ to $1100^{\circ} \mathrm{C}$ at $50^{\circ} \mathrm{C}$ intervals. After the blackbody temperature was allowed to stabilize at each step, the output of the detector/amplifier combinations was measured on an oscilloscope. Separate measurements were taken, each with one of two filters having center transmission wavelengths of $1.76 \mu \mathrm{m}$ and $4.50 \mu \mathrm{m}$ for each detector. These filters were chosen because the wavelengths in question have importance in mid-IR pyrometer experimentation. Therefore, the detectors' response at these wavelengths would be directly applicable to the design of the final, compact, solid-state pyrometer. The oscilloscope signal appeared as a roughly square-wave pulse train that increased in amplitude as temperature increased, until detector saturation occurred (well before $1100^{\circ} \mathrm{C}$ when no filters were used). We then saved the scope data to spreadsheet files and plotted graphs (see Figs. 5-8).

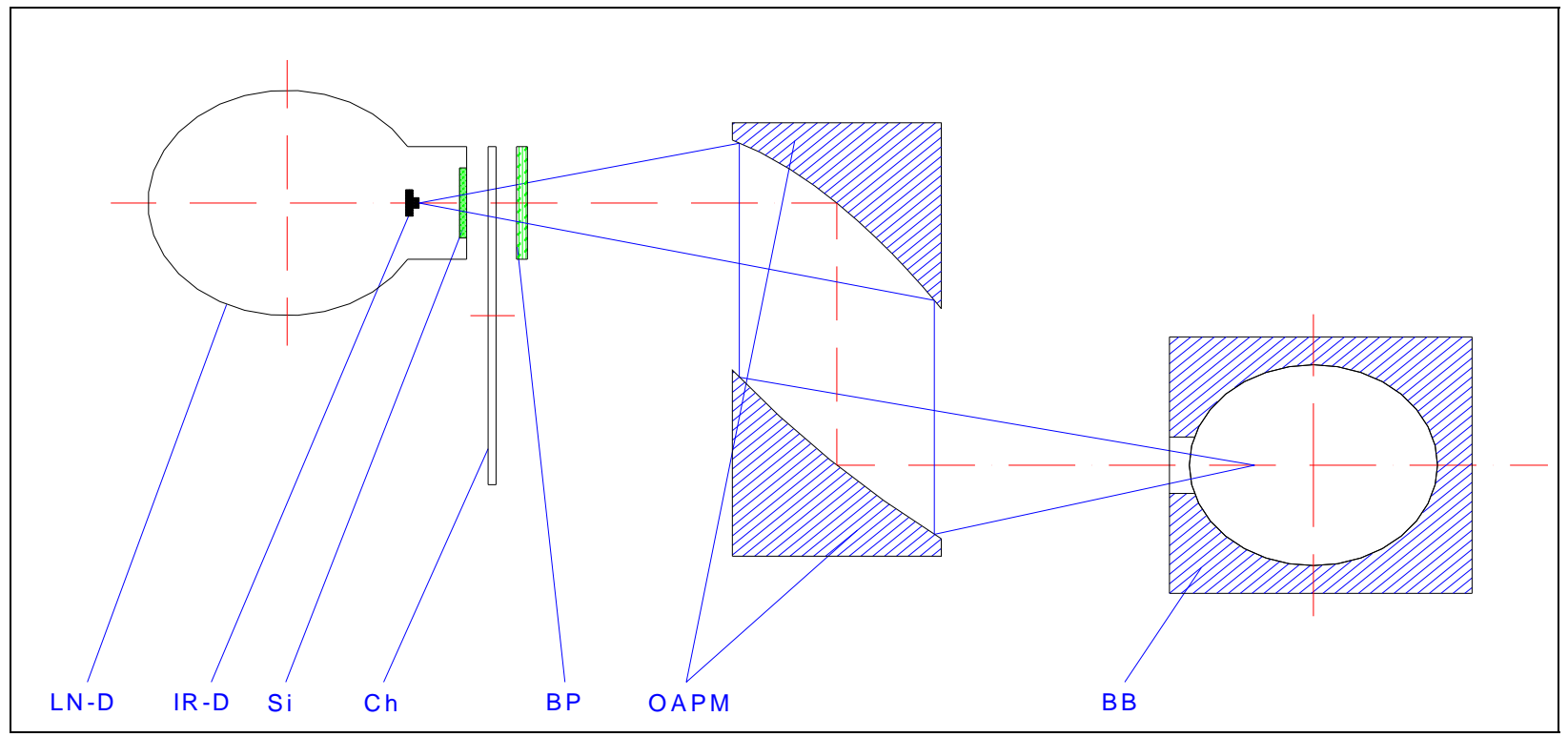

Fig. 3. Experimental setup used for detector comparison. BB, blackbody radiator; OAPM, off-axis parabolic mirrors; BP, bandpass filter; $\mathrm{Ch}$, chopper wheel; Si, silicon window; IR-D, detector; LN-D, liquid nitrogen dewar. 


\subsection{Amplifier comparison}

The amplifiers used to enhance detector signals require a two-stage design: an initial transimpedance amplifier that allows for maximum bandwidth and power transfer, and a final voltage amplifier (usually with a fixed gain). Three different amplifiers made by the Perry Amplifier Company ${ }^{8}$ were compared: one used in the existing pyrometer, one comparable but with greater bandwidth, and one with capacitive coupling between the transimpedance and output stages.

In order to examine the recovery characteristics of each amplifier without the accompanied detector response to taint the results, current was injected into the amplifiers both directly and through an RC network used to simulate output impedance (see Figure 4). The current source was a pulse generator with high-impedance outputs. The amplifiers' output impedance was matched to $50 \Omega$. Pulse amplitude was increased until saturation occurred, then taken beyond this point to examine the effects. Pulse width was varied as well, and the results were documented.

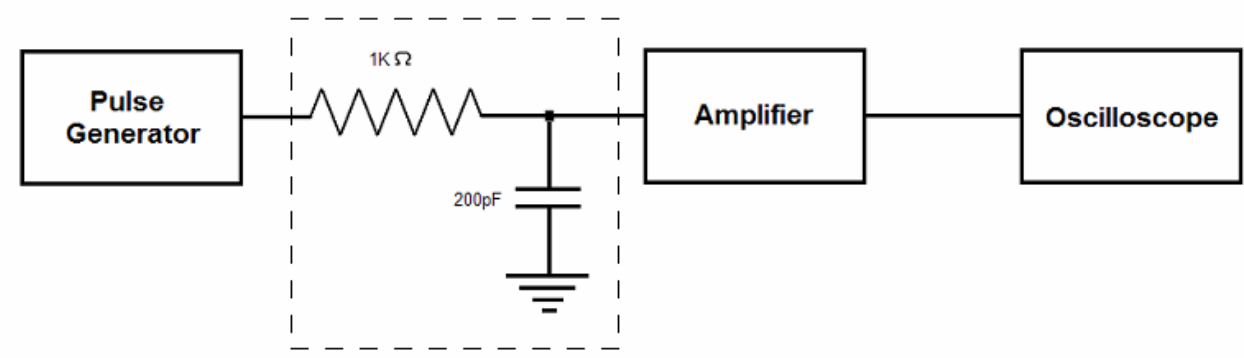

Fig. 4. Amplifier test setup with simulated detector source (current injector).

\section{COMPARISON DATA}

\subsection{Detector comparison data}

Figure 5 shows the relative speed of each detector. All detectors were measured with the same pulse width and amplitude from a $1.55-\mu \mathrm{m}$ laser diode source. The same amplifier was used for each. Clearly the Judson InSb detector had the fastest response of the three detectors evaluated. The elongated signal decay of the Fermionics MCT may derive from the detector's inclusion of a built-in, reverse-protection diode that may add stray capacitance.

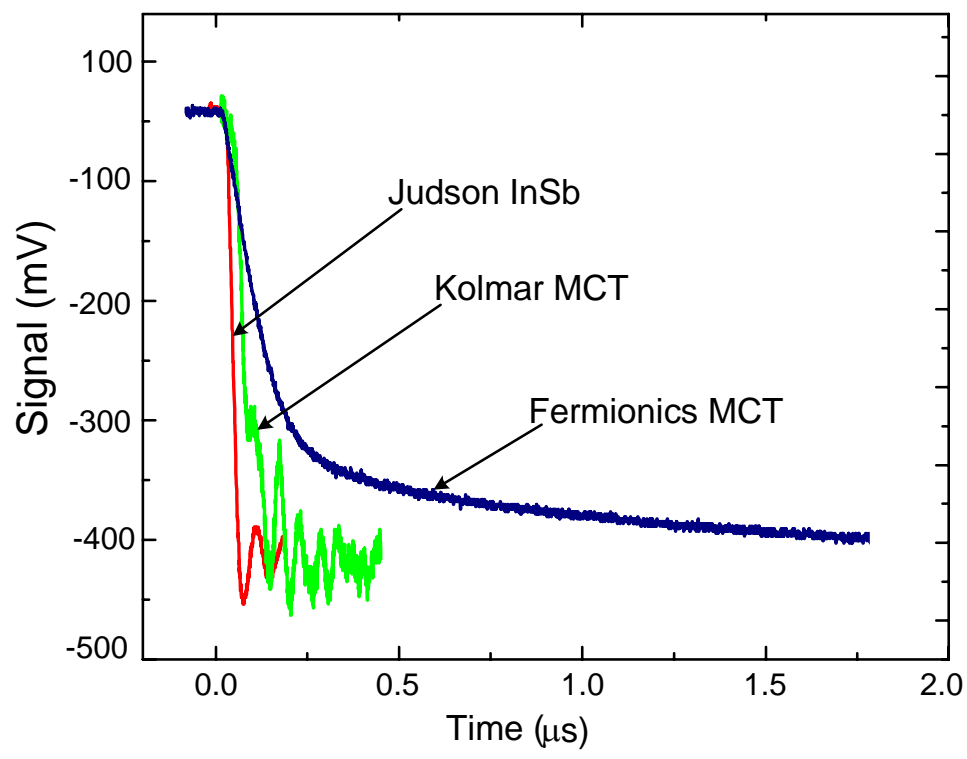

Fig. 5. Rise-time (bandwidth) measurements of detectors. 
Figure 6 plots the signal output from the detectors (through the same amplifier) versus the temperature of the target source (a calibrated blackbody radiator) over the entire range of temperatures (from $100^{\circ} \mathrm{C}$ to $1100^{\circ} \mathrm{C}$ ). Measurements were taken with both $1.76-\mu \mathrm{m}$ and $4.50-\mu \mathrm{m}$ filters. However, a plot for the Kolmar MCT detector with the $1.76-\mu \mathrm{m}$ filter does not exist because measurements were taken after those taken for the $4.50-\mu \mathrm{m}$ filter, and the detector had been returned to Sandia National Laboratories. The plots effectively show the detectors' dynamic range, with the Judson InSb having the greatest range. Both the Judson InSb and the Kolmar MCT saturated at about $9.5 \mathrm{~V}$, which far surpassed the Fermionics MCT's saturation point of approximately $3.5 \mathrm{~V}$. However, the Judson InSb was sensitive to temperatures about $100^{\circ} \mathrm{C}$ lower than that of the Kolmar MCT, as shown in the plots with the $4.50-\mu \mathrm{m}$ filter.

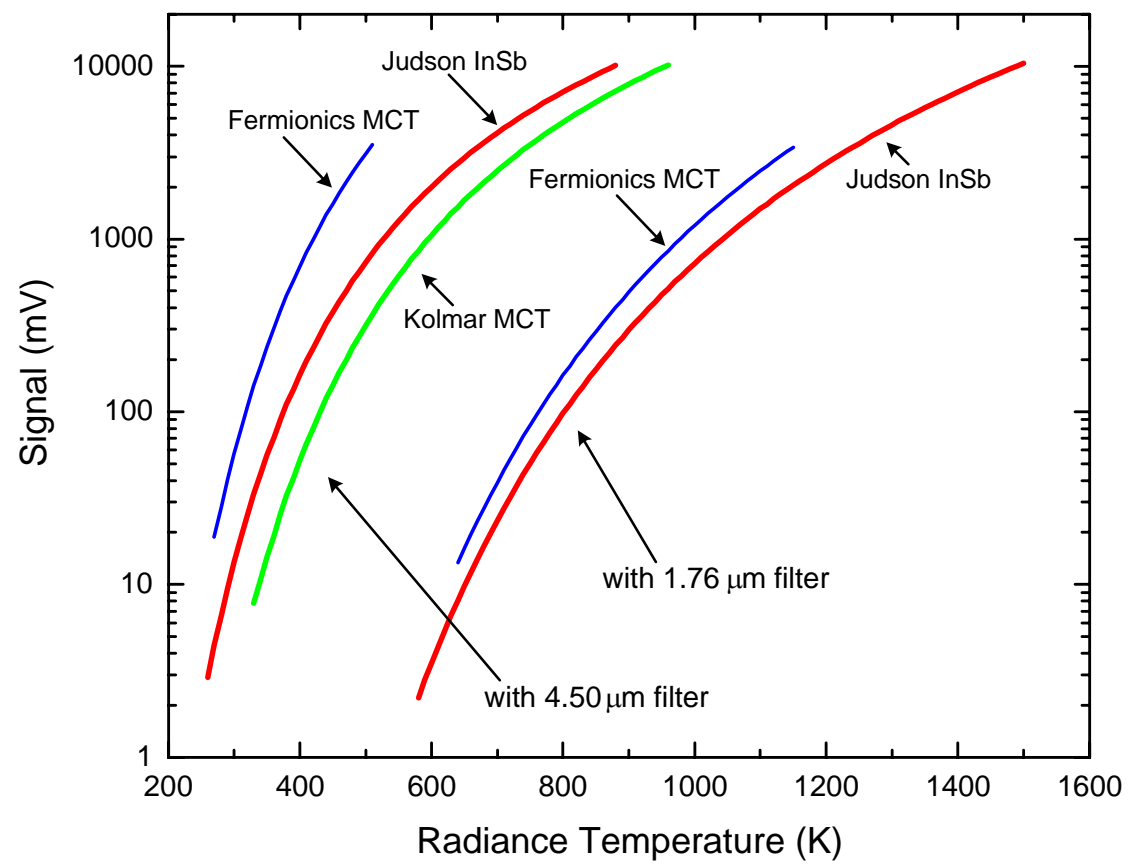

Fig. 6. Dynamic range measurements of detectors.

The top graphs in Figure 7 show inherent detector noise with no incident radiation on their surface ("dark noise"), and the bottom graphs show the effective temperature error caused by that noise. The bottom plots were made from the outputs of both detectors in unsaturated states with continuous radiant input. No comparison was made to the Kolmar MCT for the same reason as cited above re: Figure 6. Clearly, the high bandwidth and dynamic range of the Judson InSb contribute to a noisier environment.

Figure 8 illustrates the "noise equivalent temperature," or the temperature error caused by detector noise throughout the range of measured temperatures. Again, the Fermionics MCT is less noisy than the Judson InSb. Note that the signal-to-noise ratio increases with temperature as radiance (input signal) increases and noise remains constant. 


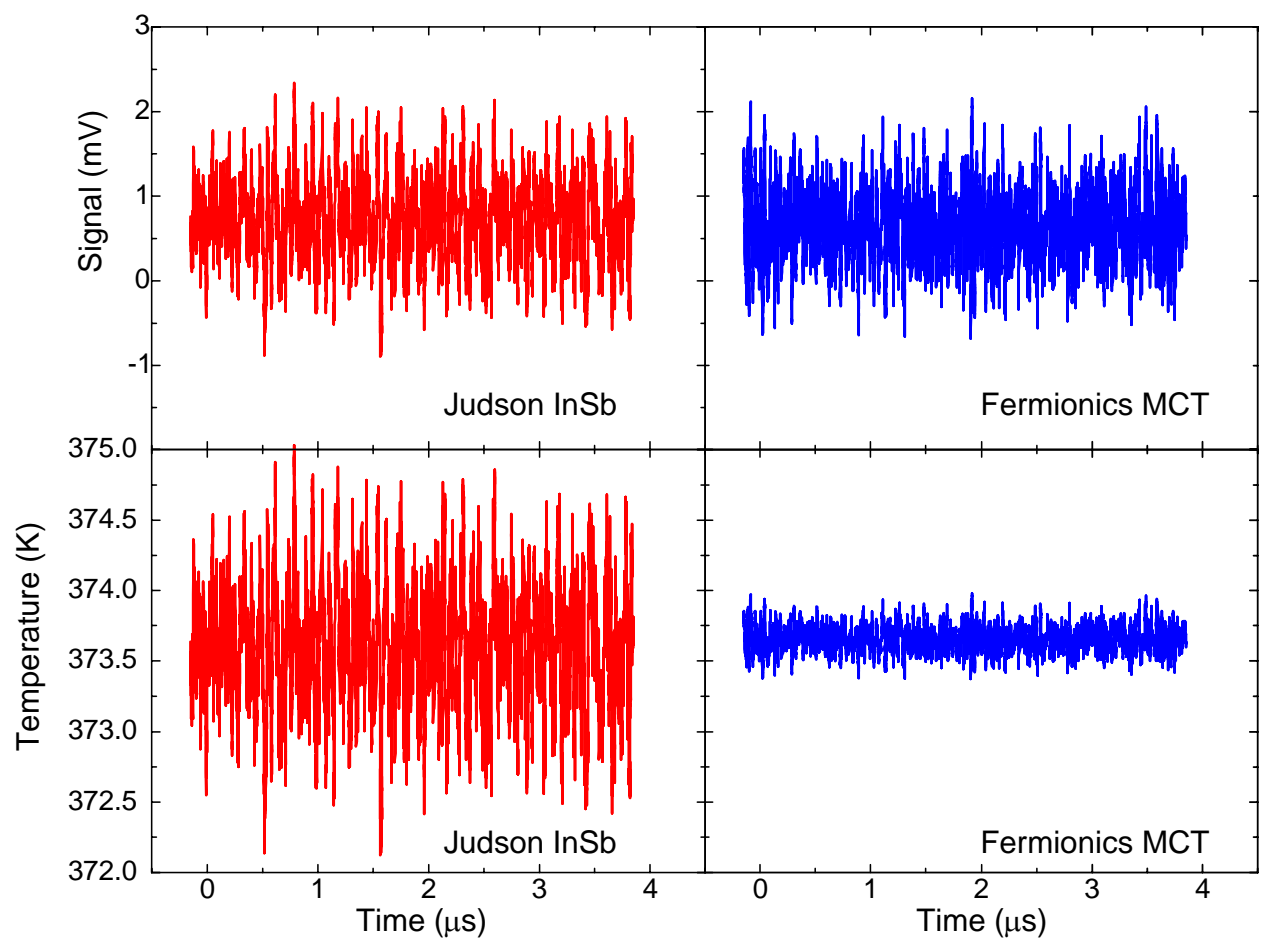

Fig. 7. Dark noise measurements (top) and corresponding temperature "noise" error (bottom).

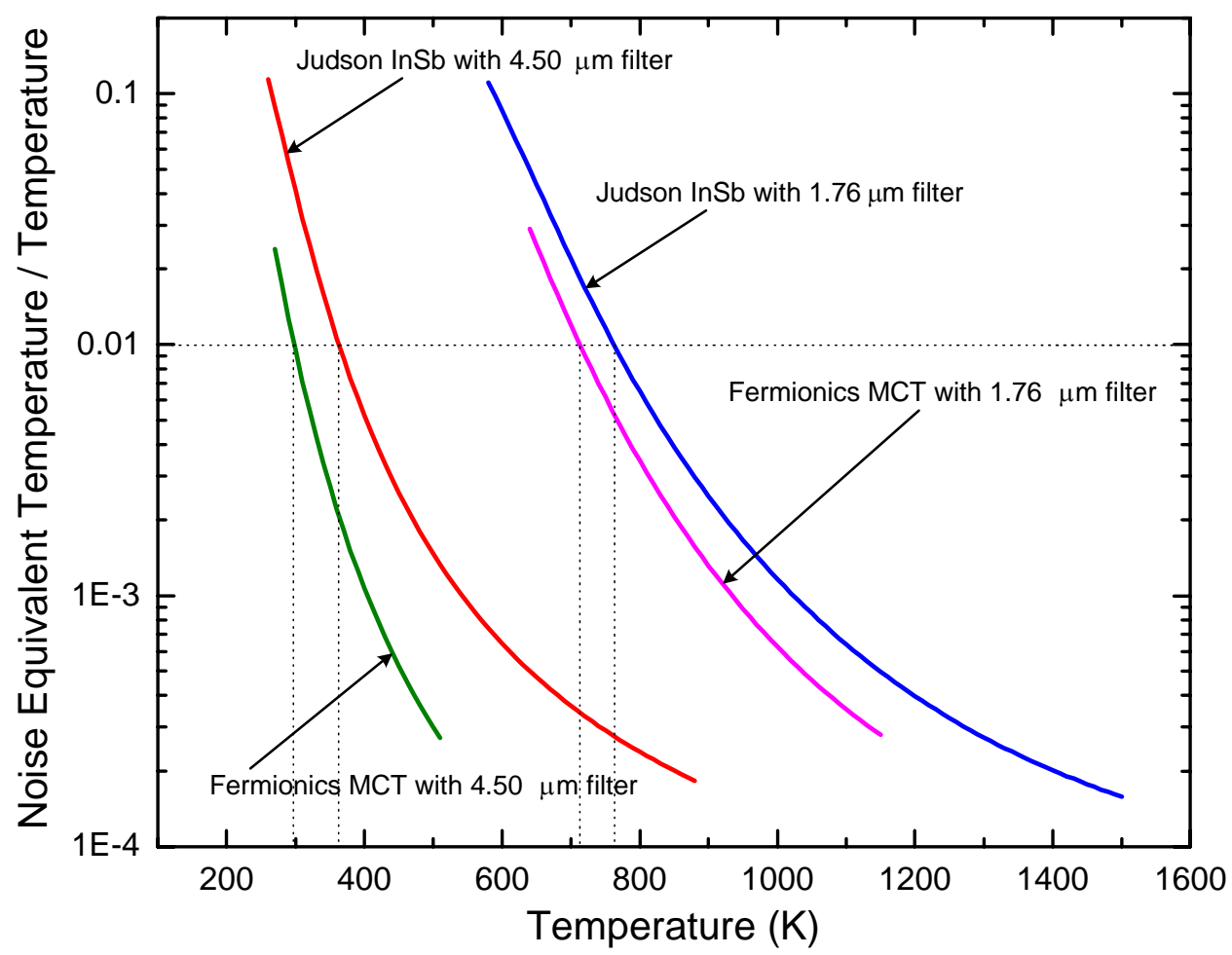

Fig. 8. Noise equivalent temperature. 


\subsection{Amplifier comparison data}

No significant effect was seen on recovery time and bandwidth, either with or without the RC impedance-matching network. Therefore, we did not compare its effects in this report. All measurements shown were made with the RC network.

Figure 9 shows the relative speed of each amplifier using a fast rise-time pulse generator. The New Amplifier \#1 shows a slight improvement over the Old Amplifier. The signal from New Amplifier \#2 is inverted by its output stage electronics. It shows a slow roll-off but no overshoot.

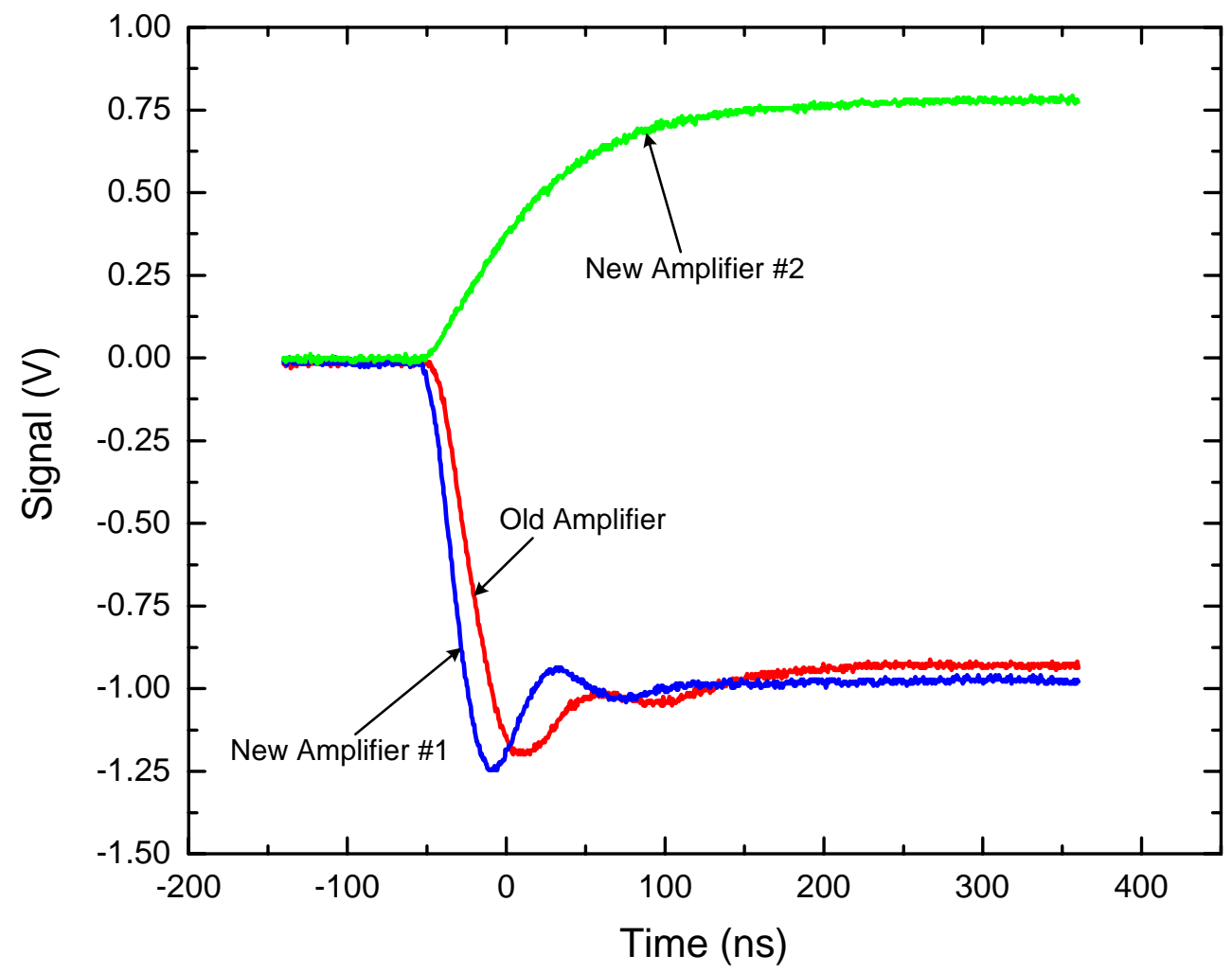

Fig. 9. Rise-time (bandwidth) measurements of amplifiers.

Figure 10 plots the saturation and recovery characteristics of each amplifier. The blue waveform in each plot is the injected pulse input. The New Amplifier \#1 shows a marked improvement in recovery time over the Old Amplifier. New Amplifier \#2 shows the best recovery time and no overshoot, though it did exhibit nonlinear characteristics. The waveform for New Amplifier \#2 has been inverted in software for comparison purposes.

The amplifiers were taken well beyond initial saturation to examine their recovery characteristics. An increase in the input pulse width and/or pulse amplitude increased recovery time. Figure 11 illustrates the effect of total charge (current $\times$ time) on amplifier recovery time between the Old Amplifier and New Amplifier \#1. The new amplifier design shows a faster recovery from excess input charge conditions. 


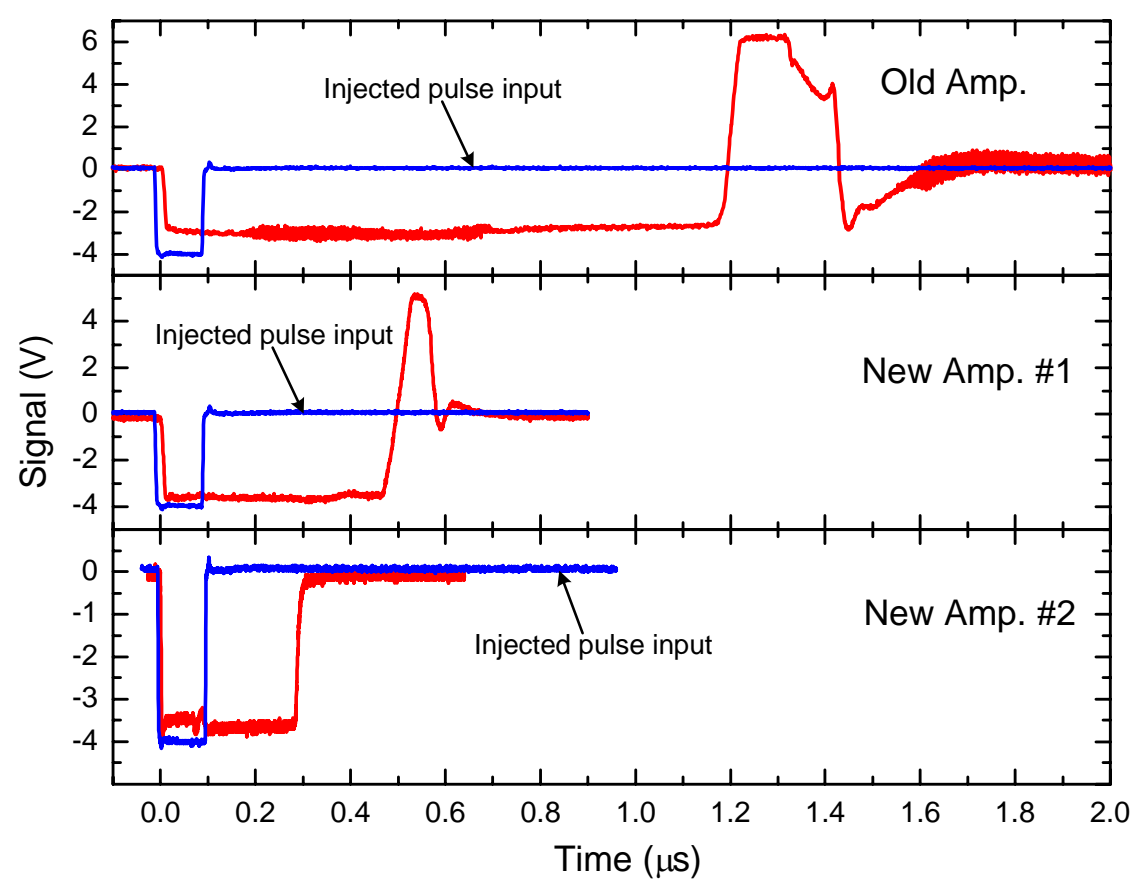

Fig. 10. Saturation characteristics of each amplifier.

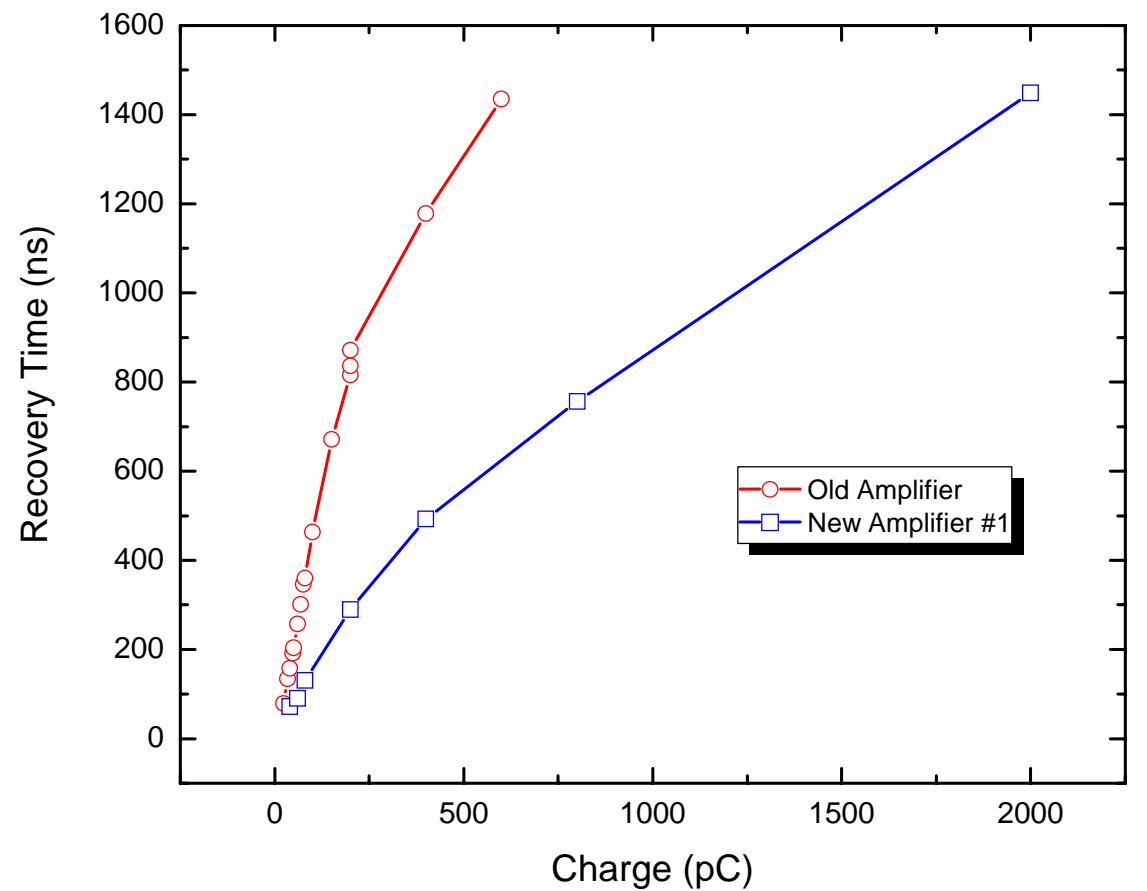

Fig. 11. Amplifier recovery time using direct current injection. 
DOE/NV/11718-1101

\section{CONCLUSIONS}

\subsection{Detectors}

For high-speed applications, the Judson InSb detector is the clear choice. It exhibits the fastest response, the best sensitivity, and the greatest dynamic range. However, where noise is the greatest issue, the Kolmar MCT could be the best option, especially for low-temperature measurements in which noise can be the dominant signal. We discussed the possibility of adjusting an MCT's detector chemistry (semiconductor composition) to increase its response time and peak wavelength sensitivity, ${ }^{5}$ thus effectively yielding the best of both worlds.

\subsection{Amplifiers}

The new amplifier designs recovered faster from saturation than the old one. However, they were still too slow to recover from the type of major shock breakout flash that can occur. One possible solution is the use of a peak detection "clipper" circuit that would either shunt excess charge or effectively "crowbar" transimpedance amplifier output before critical saturation could occur.

\section{REFERENCES}

1. V.W. Yuan, J.D. Bowman, D.J. Funk, G.L. Morgan, J.P. Quintana, R.L. Rabie, C.E. Ragan, H.L. Stacy, “Shock Temperature Measurements Using Neutron Resonance Spectroscopy,” Phys. Rev. Lett. 94, 125504, 2005.

2. K. Boboridis, A.W. Obst, "A High-Speed Four-Channel Infrared Pyrometer," in Temperature: Its Measurement and Control in Science and Industry, 7, Part 2, 759, AIP, New York, 2003.

3. D.P. DeWitt, G.D. Nutter, Theory and Practice of Radiation Thermometry, John Wiley \& Sons, New York, 1988.

4. A. Seifter, M. Grover, D.B. Holtkamp, J.R. Payton, P. Rodriguez, D. Turley, A.W. Obst, "Low-Temperature Measurements on Shock-Loaded Tin," Proceedings of High Speed Photography and Photonics, 20-24 September 2004, ed. D.L. Paisley et al., 5580, paper 124, Alexandria, VA, 2005.

5. Judson Technologies, LLC, 221 Commerce Dr., Montgomeryville, PA 18936; www.judtech.com, accessed October 12, 2004.

6. Fermionics, 4555 Runway St., Simi Valley, CA 93063; www.fermionics.com, accessed October 12, 2004.

7. Kolmar Technologies, 3 Henry Graph Rd., Unit 9, Newburyport, MA 01950; www.kolmartech.com, accessed October 12, 2004.

8. Perry Amplifier, 138 Fuller St., Brookline, MA 02446; perryampco@yahoo.com, accessed October 12, 2004.

Copyright. This manuscript has been authored by Bechtel Nevada under Contract No. DE-AC08-96NV11718 with the U.S. Department of Energy. The United States Government retains and the publisher, by accepting the article for publication, acknowledges that the United States Government retains a non-exclusive, paid-up, irrevocable, world-wide license to publish or reproduce the published form of this manuscript, or allow others to do so, for United States Government purposes.

Disclaimer. This report was prepared as an account of work sponsored by an agency of the U.S. Government. Neither the U.S. Government nor any agency thereof, nor any of their employees, nor any of their contractors, subcontractors or their employees, makes any warranty or representation, express or implied, or assumes any legal liability or responsibility for the accuracy, completeness, or usefulness of any information, apparatus, product, or process disclosed, or represents that its use would not infringe privately own rights. Reference herein to any specific commercial product, process, or service by trade name, trademark, manufacturer, or otherwise, does not necessarily constitute or imply its endorsement, recommendation, or favoring by the U.S. Government or any agency thereof. The views and opinions of authors expressed herein do not necessarily state or reflect those of the U.S. Government or any agency thereof. Paper number DOE/NV/11718-1101. 